\title{
A Case of Successful Placement of a Fully Covered Metallic Stent for Hemobilia Secondary to Hepatocellular Carcinoma with Bile Duct Invasion
}

\author{
Yoshiaki Kawaguchi Masami Ogawa Atsuko Maruno \\ Hiroyuki Ito Tetsuya Mine \\ Department of Gastroenterology, Tokai University School of Medicine, \\ Isehara, Japan
}

\section{Key Words}

Hemobilia $\cdot$ Hepatocellular carcinoma - Bile duct invasion - Fully covered metallic stent $\cdot$ Hemostasis

\begin{abstract}
Hemobilia represents gastrointestinal bleeding that develops as a result of communication between blood vessels and the biliary tract, which causes the blood to reach the duodenal papilla. It is characterized by biliary colic as the initial symptom, and the complications of cholangitis, obstructive jaundice and/or anemia. In general, definitive diagnosis is made by esophagogastroduodenoscopy which confirms bleeding from the duodenal papilla.

Abdominal US and abdominal enhanced CT are performed to identify the source of the bleeding, as well as ERCP for biliary drainage to control the comorbid cholangitis. If active hemorrhage accompanied by worsening of the anemia is suspected, abdominal angiography is performed to selectively image the hepatic artery. Then, embolization of the culprit vessel is recommended. In our patients with difficult hemostasis, because of the direct compression hemostasis to the tumor site achieved with the fully covered metallic stent and secondary compression hemostasis due to blood clots, the bleeding could be controlled.
\end{abstract}

\section{Case Report}

A 63-year-old man, who had been under treatment for alcoholic cirrhosis and hepatocellular carcinoma, was admitted emergently to our hospital because of black stool. A blood test revealed increase of the serum levels of hepatobiliary enzymes, jaundice, and anemia. An urgent upper gastrointestinal endoscopy was performed, which did not reveal the bleeding source. Lower 
gastrointestinal endoscopy and capsule endoscopy were also performed; however, the site of bleed ing could not be identified. Based on the findings of abdominal ultrasonography (US) and abdominal enhanced computed tomography (CT), hepatocellular carcinoma with invasion of the left intrahepatic bile duct and biliary duct dilatation was suspected ( fig. 1a). Bleeding scintigraphy suggested bleeding from the left lobe of the liver. Endoscopic retrograde cholangiopancreatography (ERCP) revealed a filling defect in the area extending from the beginning of the left intrahepatic bile duct to the hepatic portal region, confirming bleeding from the duodenal papilla, which led to the diagnosis of hemobilia (fig. 1b, c). Hepatocellular carcinoma was detected by brushing cytology of the filling defect area; thus, the hemobilia was determined to be caused by hepatocellular carcinoma invasion of the bile duct. Because it was difficult to stop the bleeding by transcatheter arterial embolization (TAE), partly as a result of repeated sessions of transcatheter arterial chemoembolization (TACE) which the patient had previously undergone, we placed a fully covered metallic stent that extended from the common bile duct to the right intrahepatic bile duct branch, compressing the filling defect area of the common bile duct. During the 2 months until the patient died of cancer, the gastrointestinal bleeding and anemia could be controlled ( $\underline{\text { fig. } 2}$ ), without complicating cholangitis.

\section{Discussion}

Hemobilia represents gastrointestinal bleeding that develops as a result of communication between blood vessels and the biliary tract, which causes the blood to reach the duodenal papilla. It is characterized by biliary colic as the initial symptom, and the complications of cholangitis, obstructive jaundice and/or anemia.

The causes of hemobilia include inflammation, calculus, tumor, injury, blood vessel disorder, and surgery. In regard to tumor causes, hemobilia is often caused by invasion of the bile duct by hepatocellular carcinoma, a hypervascular tumor. TACE or radiofrequency ablation therapy is widely performed for the treatment of hepatocellular carcinoma at present. Recurrence or progression of cancer or repeated treatment of the cancer using the aforementioned modalities may cause invasion of the biliary tract by the hepatocellular carcinoma; sometimes, arterial injury due to treatment may cause formation of a pseudoaneurysm. Our patient had undergone repeated sessions of TACE for the hepatocellular carcinoma, which had resulted in invasion of the biliary tract by the liver cancer.

In general, definitive diagnosis is made by esophagogastroduodenoscopy, which confirms bleeding from the duodenal papilla. Abdominal US and abdominal enhanced CT are performed to identify the source of the bleeding, as well as ERCP for biliary drainage to control the comorbid cholangitis. In the present case, cholangiography allowed detection of the bleeding from the duodenal papilla for the first time. Cholangiography revealed the blood clot as a mobile filling defect in the biliary tract. In general, if biliary tract drainage is required, a biliary tract drainage tube is placed after the cholangiography. However, because the tube is prone to blockage by blood clots, a cleanable external fistula tube is recommended rather than an internal fistula tube.

If active hemorrhage accompanied by worsening of the anemia is suspected, abdominal angiography is performed to selectively image the hepatic artery. Then, definitive diagnosis can be made if the culprit vessel is identified and extravasation into the biliary tract is demonstrated. Then, embolization of the culprit vessel is recommended because it is less invasive and associated with a high likelihood of hemostasis [1-6]. However, since this patient had undergone repeated sessions of TACE, it was difficult to approach the culprit vessel and stop the bleeding. In cases 
where even TAE fails to establish hemostasis, surgical therapy, such as hepatic artery ligation or hepatectomy, is required. However, since this patient had advanced hepatocellular carcinoma and cirrhosis of the liver, such highly invasive surgical therapy was not opted for.

In patients with difficult hemostasis, as in this patient, there are a few reports of cases treated by placement of a covered metallic stent in the biliary tract $[7,8]$. In general, placement of a covered metallic stent in the hepatic hilar bile duct may cause cholangitis due to bile duct branch occlusion, and is not recommended. In this case, however, we placed a fully covered metallic stent in the hepatic hilar bile duct to control hemobilia secondary to bile duct invasion caused by hepatocellular carcinoma, because of the difficulty in establishing hemostasis and the advanced terminal cancer. In the end, because of the direct compression hemostasis to the tumor site (common bile duct) achieved with the fully covered metallic stent and secondary compression hemostasis due to blood clots (left intrahepatic bile duct), the bleeding could be controlled. Although cholangitis did not occur in our case, measures such as placement of an endoscopic nasobiliary drainage tube into the stent-obstructed bile duct may need to be considered for the control of potentially complicating cholangitis. Thus, placement of a covered metallic stent would seem to be a valid option that can be used for the treatment of hemobilia in patients with advanced cancer and difficulty in the establishment of hemostasis. 

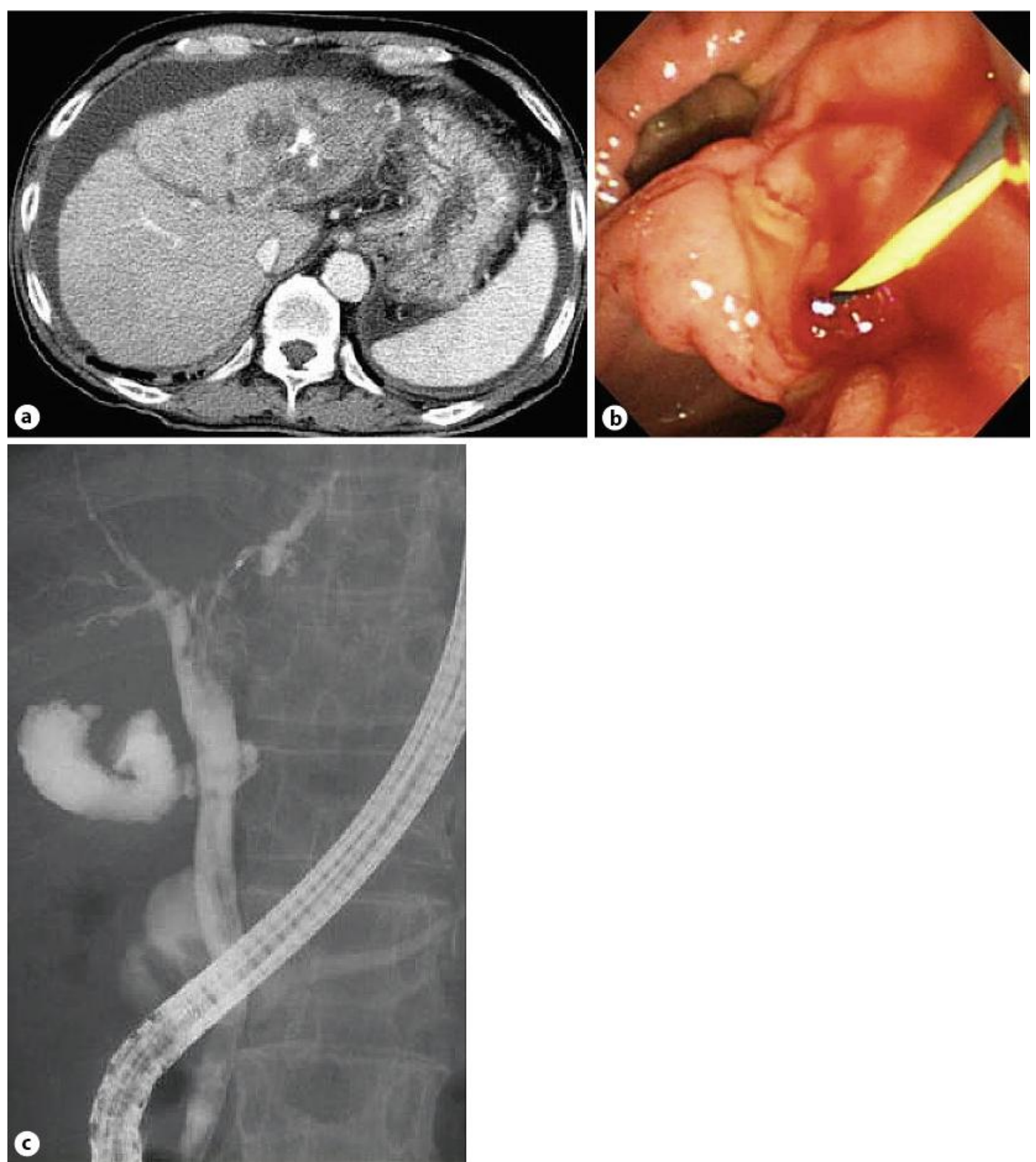

Fig. 1. a Abdominal enhanced CT shows features suggestive of an invasive hepatocellular carcinoma and dilatation of the left intrahepatic bile duct. b, c Cholangiography shows a defect suggestive of a clot or tumor in the left bile duct (b). ERCP shows bleeding (a). 


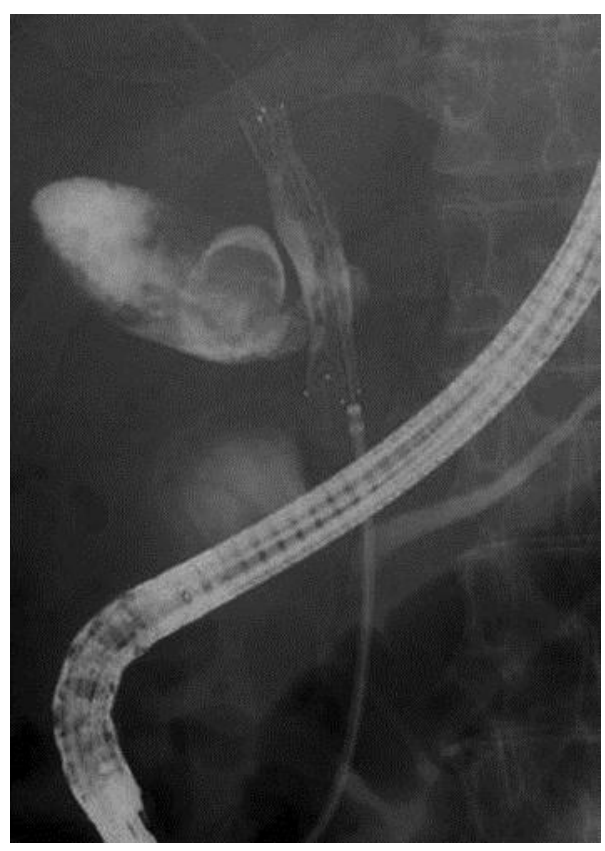

Fig. 2. A fully covered metallic stent was placed in the common bile duct covering the left intrahepatic bile duct.

\section{References}

1 Walter JF, et al: Successful transcatheter embolic control of massive hematobilia secondary to liver biopsy. Am J Roentgenol 1976;127:847-849.

-2 Heimbach DM, et al: Treatment of traumatic hemobilia with angiographic embolization. J Trauma 1978;18:221-224.

-3 Curet P, et al: Hepatic hemobilia of traumatic or iatrogenic origin: recent advances in diagnosis and therapy, review of the literature from 1976 to 1981. World J Surg 1984;8:2-8.

4 Okazaki M, et al: Angiographic management of massive hemobilia due to iatrogenic trauma. Gastrointest Radiol 1991;16:205-211.

5 Savader SJ, et al: Hemobilia after percutaneous transhepatic biliary drainage: treatment with transcatheter embolotherapy. JVIR 1992;3:345-352.

-6 Livraghi T, Solbiati L, Meloni MF, et al: Treatment of focal liver tumors with percutaneous radiofrequency ablation: complications encountered in a multicenter study. Radiology 2003;226:441451.

-7 Rerknimitr R, Kongkam P, Kullavanijaya P: Treatment of tumor associated hemobilia with a partially covered metallic stent. Endoscopy 2007;39:E225.

-8 Vanangunas A, Ehrenpreis E: Endoscopic evacuation of hematobilia induced by large bore selfexpanding biliary mesh stent. Gastrointest Endosc 1991;37:101-103. 